\title{
The Effect of Ageing Process on the Electrogyration of Deuterated Triglycine Sulphate Crystals
}

\author{
M. Koralewski, J. Stankowska and S. HabryŁo \\ Institute of Physics, A. Mickiewicz University \\ Umultowska 85, 61-614 Poznań, Poland
}

(Received June 18, 2001; revised version November 19, 2001)

\begin{abstract}
The paper reports results of a study of optical activity and ferroelectric properties of rejuvenated and aged deuterated triglycine sulphate crystals. A possibility of monitoring of the ferroelectric crystal ageing process by optical measurements was proved. A linear relation between optical rotatory power $\rho$ and spontaneous polarization $P_{\mathrm{s}}$ was confirmed for aged and rejuvenated deuterated triglycine sulphate crystals.
\end{abstract}

PACS numbers: 77.80.Bh, 78.20.Ek

\section{Introduction}

The optical activity (OA) of triglycine sulphate crystal (TGS) along the optical axis ( $k \|$ optical axis - isotropic direction) was for the first time observed by Hermelbracht and Unruh [1] in 1970 and shortly after their report confirmed by Vlokh et al. [2]. These authors have proved a linear relationship between the optical rotatory power $(\rho)$ and spontaneous polarization $\left(P_{\mathrm{s}}\right)$. Later our works $[3,4]$ provided experimental confirmation of the enantiomorphic character of domains in the ferroelectric TGS crystals on the basis of the measurements of the hysteresis loop of the optical rotatory power in the direction of the optical axis. The results were the first experimental verification of the predictions of Shuvalov et al. [5]. The enantiomorphic character of TGS domains was confirmed by Kobayashi et al. [6] for the anisotropic direction ( $k \| y$ - anisotropic direction). For the last two decades the optical activity of TGS type crystals has been studied in detail by several authors [6-9] and a correlation between the electrogyration and ferroelectric properties has been well documented. A linear relationship between $\rho$ and $P_{\mathrm{s}}$ as 
well as a possibility of observation of the hysteresis loop of the optical rotatory power indicates that optical activity measurements can be successfully used to study ferroelectric crystals.

One of the most important problems studied in ferroelectric crystals is their ageing manifested as stabilization of spontaneous polarization. This process leads to time changes of certain physical parameters such as $P_{\mathrm{s}}, \varepsilon, E_{\mathrm{c}}$. The ageing of TGS crystals has been mainly studied by dielectric methods [10-12], whereas the optical aspects have been rather sporadically a subject of concern.

The aim of the paper is to show that the process of ageing of the ferroelectrics crystals can be monitored by measurements of the electrogyration effect. The measurements reported have been performed for a deuterated TGS (DTGS) crystal.

\section{Experimental}

DTGS crystals were grown by the dynamical method from a saturated heavy water solution of DTGS. The degree of deuteration $(X)$ of TGS crystals was determined by measurements of the Curie temperature $T_{\mathrm{C}}$ and on the grounds of paper of Březina and Smutny where a relation between $T_{\mathrm{C}}$ and the deuteration degree can be found [13]. The most deuterated TGS crystal $(X \approx 90 \%)$ was chosen for measurements. The samples were cut out from a bulk crystal perpendicularly to the ferroelectric axis and the optical axis (at $\lambda=632.8 \mathrm{~nm}$ ) for dielectric and optical measurements, respectively.

OA measurement procedure and experimental set up was the same as described previously $[3,4]$. Both samples - for dielectric and OA measurements were rejuvenated in the same way (without electrodes) by annealing for 24 hours at $363 \mathrm{~K}$.

\section{Results and discussion}

Spontaneous polarization of the DTGS crystal studied has been determined for rejuvenated and aged samples, Fig. 1. The value of $P_{\mathrm{s}}$ of the rejuvenated sample is higher as expected in view of earlier reports [12]. The spontaneous polarization and the temperature dependences of the optical rotatory power are shown in Fig. 2. The temperature dependence of the optical rotatory power above the Curie point is constant and equal to zero but below the $T_{\mathrm{C}}$ this dependence shows a similar behaviour as $P_{\mathrm{s}}$ vs. $T$. A least squares linear regression method was used to check the proportionality between $\rho$ and $P_{\mathrm{s}}$. A linear relationship between $\rho$ and $P_{\mathrm{s}}$ has been found (see Fig. 2), similarly as for other crystals from the TGS family $[1-4,14]$ :

$$
\rho=\delta P_{\mathrm{s}},
$$

where $\delta$ is the proportionality coefficient. 


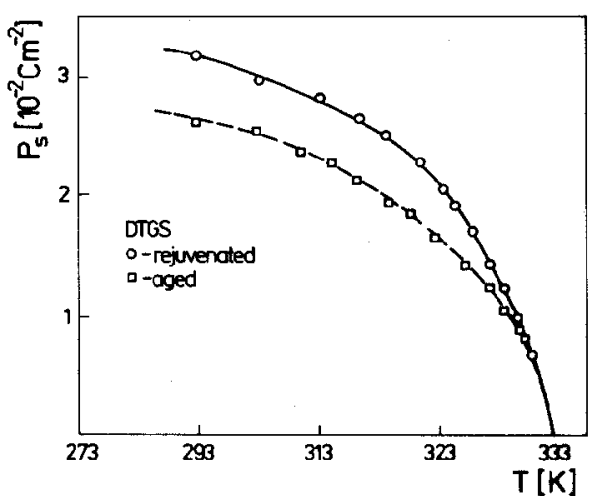

Fig. 1. Temperature dependence of spontaneous polarization $P_{\mathrm{s}}$ for aged $(\square)$ and rejuvenated (o) DTGS crystals.

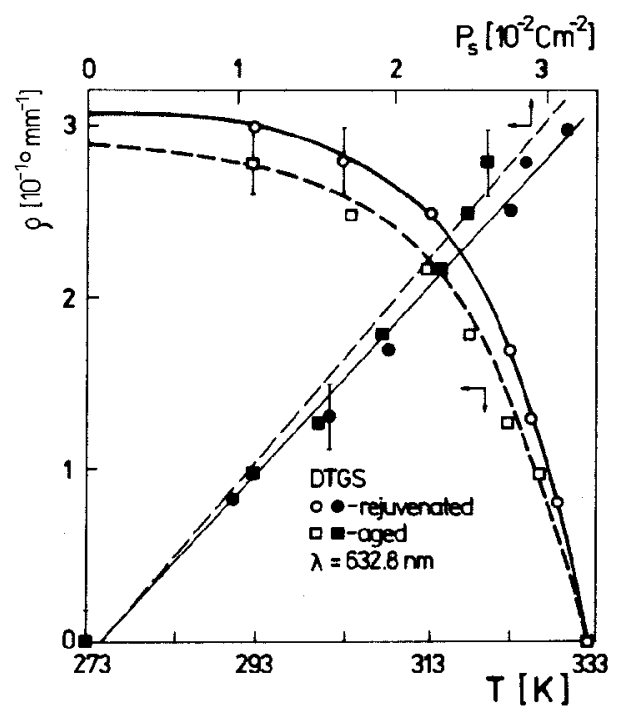

Fig. 2. The optical rotatory power $\rho$ of aged $(\boldsymbol{\bullet}, \square)$ and rejuvenated $(\circ, \bullet)$ DTGS crystals versus temperature $(\circ, \square)$ and spontaneous polarization $P_{\mathrm{s}}(\boldsymbol{\bullet}, \bullet)(\lambda=632.8 \mathrm{~nm})$.

The small negative value of intercept may be noticed (see Fig. 2) but it is below error bar and may be neglected. The coefficient $\delta$ was calculated as slope of $\rho$ vs. $P_{\mathrm{s}}$ and is given in Table. Slightly higher value of the coefficient $\delta$ for aged crystal of DTGS in comparison to rejuvenated one may be seen (Table), it suggests that the value of this coefficient may be useful as some indication of aged state of ferroelectric crystals. The values of $\delta$ for rejuvenated and aged DTGS are higher than those for TGS crystal [1-3].

The effect of ageing on electrogyration phenomenon is the most pronounced in the hysteresis loop of the optical rotatory power recorded for aged and rejuve- 
TABLE

Some parameters for aged and rejuvenated samples of DTGS crystal $(T=293 \mathrm{~K}, \lambda=$ $632.8 \mathrm{~nm})$.

\begin{tabular}{l|c|c}
\hline \hline \multirow{2}{*}{ Parameter } & \multicolumn{2}{|c}{ DTGS } \\
\cline { 2 - 3 } & aged & rejuvenated \\
\hline$T_{\mathrm{C}}[\mathrm{K}]$ & 332.7 & 332.7 \\
$P_{\mathrm{S}}\left[10^{-2} \mathrm{C} \mathrm{m}^{-2}\right]$ & 2.6 & 3.2 \\
$\rho\left[10^{-1} \circ \mathrm{mm}^{-1}\right]$ & 2.8 & 3.0 \\
$\delta\left[10^{2} \mathrm{~m} \mathrm{C}^{-1}\right]$ & 1.85 & 1.63 \\
$K_{1}^{\prime}$ & 4.33 & 4.40 \\
$K_{1}^{\prime \prime}$ & -4.34 & -4.41 \\
$\lambda_{1}[\mu \mathrm{m}]$ & 0.105 & 0.105
\end{tabular}

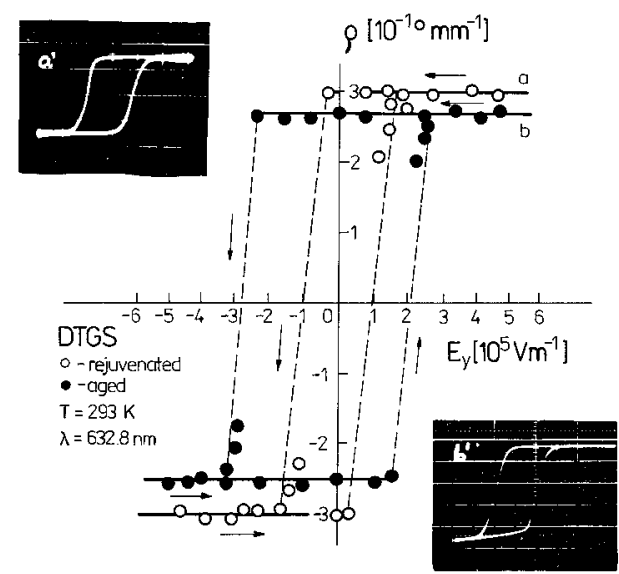

Fig. 3. Hysteresis loops of the optical rotatory power $(\mathrm{a}, \mathrm{b})$ and ferroelectric hysteresis loops $\left(\mathrm{a}^{\prime}, \mathrm{b}^{\prime}\right)$ for aged $\left(\mathrm{b}, \mathrm{b}^{\prime}\right)$ and rejuvenated $\left(\mathrm{a}, \mathrm{a}^{\prime}\right)$ DTGS crystals $(T=293 \mathrm{~K}$ and $\lambda=632.8 \mathrm{~nm}$; the value of the measuring field intensity is different for the cases shown in parts $\left(\mathrm{a}^{\prime}\right)$ and $\left.\left(\mathrm{b}^{\prime}\right)\right)$.

nated DTGS, shown in Fig. 3. The aged samples reveal unipolarity clearly marked in the two types of hysteresis loops, Fig. 3b, $\mathrm{b}^{\prime}$. The process of rejuvenation leads to symmetrization of the two types of hysteresis loops, Fig. 3a, $\mathrm{a}^{\prime}$. The unipolarity of the loops is related to the directional polarization appearing in aged ferroelectric crystals or in the crystals defected by admixtures of foreign ions or gamma or X-ray irradiation. Similar asymmetric hysteresis loops of the optical rotatory power have been observed for TGS crystals upon X-ray irradiation [14]. The unipolarity is cancelled by rejuvenation at a temperature higher than $T_{\mathrm{C}}$ (as described 
earlier) or placement of the crystal in a constant electric field of appropriate value and direction, the so-called bias field $E_{\mathrm{b}}$. The value of $E_{\mathrm{b}}$ can be determined from the ferroelectric loops or electrogyration loops. For the DTGS crystal studied, this value is close to $3 \mathrm{kV} \mathrm{m}^{-1}$ and is low relative to the bias field observed for defected [14] or alanine admixtured crystals [15].

Dispersion of the optical rotatory power at room temperature has been studied for aged and rejuvenated samples, Fig. 4. The experimental points are well described by the Born-Vyšín equation

$$
\rho=\sum_{i} \frac{K_{i}^{\prime} \lambda^{2}}{\left(\lambda^{2}-\lambda_{i}^{2}\right)^{2}}+\sum_{i} \frac{K_{i}^{\prime \prime}}{\lambda^{2}-\lambda_{i}^{2}},
$$

where $\lambda_{i}$ is the characteristic absorption wavelength (the wavelength corresponding to the maximum of the real or effective absorption band), $K_{i}^{\prime}$ and $K_{i}^{\prime \prime}$ are constants describing the crystalline and molecular contributions. The calculations were restricted only to the case of $i=1$, and were performed by least squares method. The dispersion curves are parallel and the value of $\rho$ for an aged crystal differs by a constant from that for a rejuvenated one. The parameters of this equation are given in Table. Analysis of the parameters of Eq. (2) and dispersion curves shows that the crystalline and molecular contributions have close values but of different sign, see the values of $K$ in Table. Unfortunately, the spectral range studied is too far from the absorption bands and we are not able to identify the optical chromophores responsible for OA of the crystals and to pinpoint these which induce the change of dispersion in the aged crystals. Results of the hitherto research suggest that the main contribution to OA of TGS type crystals comes from glycine I (Gly I), because of the proportionality between $\rho$ and $P_{\mathrm{s}}$ and the

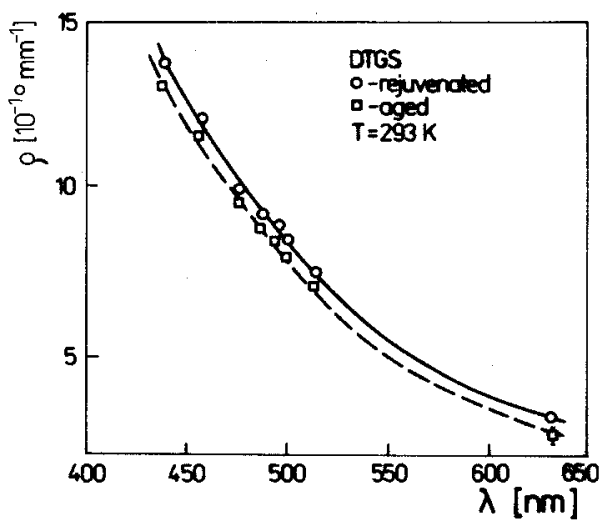

Fig. 4. Dispersion of the optical rotatory power $\rho$ of the aged $(\square)$ and rejuvenated (o) DTGS crystal ( $T=293 \mathrm{~K}$ ). The solid and dashed lines are the best fit to the experimental points calculated from Eq. (2). 
fact that the dipole moment of Gly I, responsible for the ferroelectric properties of TGS type crystals, brings the greatest contribution to $P_{\mathrm{s}}$. The electron transitions responsible for OA are localised in the carboxyl and hydroxyl groups of Gly I [16]. As a result of ageing some glycines of Gly I become fixed with respect to the plane $y=1 / 4$. This leads to a decrease in $P_{\mathrm{s}}$ and also means that the asymmetry of the chromophores will be disturbed to a lower degree or not disturbed at all. This means that the number of Gly I for which the rotatory strength $R_{k}$, expressed by a scalar product of the electric dipole transition moment $\mu_{k}^{e}$ and the magnetic dipole transition moment $\mu_{k}^{\mathrm{m}}$ as $R_{k}=\mu_{k}^{e} \mu_{k}^{\mathrm{m}} \neq 0$, decreases leading to a decrease in $R_{k}$. Consequently, the optical rotatory power of the aged crystal weakens.

In conclusion, we can say that observations of electrogyration effect can be applied for monitoring the process of ageing of TGS type crystals and other ferroelectric crystals, and that there is a strong correlation between the optical activity and ferroelectric properties of these crystals.

\section{References}

[1] K. Hermelbracht, H.G. Unruh, Z. Angew. Phys. 28, 285 (1970).

[2] O.G. Vlokh, I.W. Kutnyj, E.A. Eazko, W. Ja. Niesterienko, Izv. Akad. Nauk SSSR, Ser. Fiz. 35, 1852 (1971).

[3] S. Habryło, M. Koralewski, Acta Phys. Pol. A 60, 147 (1981).

[4] M. Koralewski, S. Habryło, Ferroelectrics 46, 13 (1982).

[5] L.A. Shuvalov, K.S. Aleksandrov, I.S. Zheludev, Kristallografia 4, 130 (1959).

[6] J. Kobayashi, Y. Uesu, H. Takehara, J. Appl. Cryst. 16, 212 (1983).

[7] J. Etxebarria, J. Ortega, T. Bręczewski, J. Phys., Condens. Matter 4, 6851 (1992).

[8] O.S. Kushnir, Y.I. Shopa, O.G. Vlokh, Ferroelectrics 143, 187 (1993).

[9] W. Kaminsky, Phase Transit. 52, 235 (1994).

[10] J. Stankowska, Ferroelectrics 22, 753 (1978).

[11] J. Stankowska, Acta Phys. Pol. XXXI, 527 (1967).

[12] J. Stankowska, A. Czarnecka, Ferroelectrics 98, 95 (1989).

[13] B. Březina, F. Smutny, Czech. J. Phys. B 18, 1924 (1968).

[14] M. Koralewski, S. Habryło, Ferroelectr. Lett. 2, 81 (1984).

[15] M. Koralewski, J. Stankowska, T. Jasinski, Jpn. J. Appl. Phys. 26, 383 (1987).

[16] M. Koralewski, Optical activity and its relation with the ferroelectric state of selected ferroelectric crystals, Ser. Phys. 73, A. Mickiewicz University Press, Poznań 2000 (in Polish). 